\title{
Application of optimization in the early stages of product development, using a small UAV case study
}

\author{
M.P. Gusev ${ }^{1}$, S.M. Nikolaev ${ }^{1}$, I.K. Uzhinsky ${ }^{1}$, D.I. Padalitsa ${ }^{1}$, and E. R. \\ Mozhenkov ${ }^{1}$ \\ Skolkovo Institute of Science and Technology, Moscow, Russia \\ m.gusev@skoltech.ru \\ https://www.skoltech.ru/en/
}

\begin{abstract}
Innovative product development involves a lot of tools, methods, and approaches to create a better product faster and satisfying customers need. Two wide spread approaches are Product Lifecycle Management (PLM) and Model-Based Systems Engineering (MBSE). However, there are not many software tools, combining methodologies PLM MBSE; one can mention LMS System Synthesis software, now is actively developing. It is used to create system models which include functional models able to describe system behavior in multiphysics domain. Therefore, when the system model of a product is multidisciplinary its not obvious what the parameters values describe the optimal state of the system. For these reasons, it is vital to apply multidisciplinary optimization techniques with the specific tools to calculate appropriate parameters of the developed system in the early stages of product development. This research is devoted to different optimization tools application, such as pSeven and Optimus Noesis, to the numerical models constituting digital twin for a small Unmanned Aerial Vehicle (UAV). The results of this research, specifically formulation of the optimization problems, detailed numerical models and the calculations results, are presented in this paper. The main idea is that optimization should be applied not as additional tool for separate numerical models, but one has to apply it as a powerful means along with PLM and MBSE tools in order to ensure that the product will meet all requirements and reduce time-to-market.
\end{abstract}

Keywords: Optimization $\cdot$ product lifecycle management $\cdot$ model-based systems engineering · small UAV · digital twin.

\section{Introduction}

Innovative product development requires a lot of software tools which allows to create a different representation of a developed product. All this software creates a lot of data which is needed to manage and Product Lifecycle Management (PLM) platform performs this function. In order to realize product to a market in a faster and proper way: PLM approach [1] combines with Model Based System Engineering (MBSE) methodology $[2,3]$. In this case, 1D numerical models 
which describe product behavior are developed in early stages of a product development, which allows to check all the requirements for the product. Also, 3D Computer-Aided Design (CAD) and Computer-Aided Engineering (CAE) models represent different level of the system, such as the geometric design, and various analytical perspectives such as performance and reliability. In order to meet all requirements in a faster and easy way, it is proposed to implement optimization approach to all numerical models and to different stages of a product development.

All stages of a product development can be represented by V-diagram [4], shown in Fig. 1



Fig. 1. V-diagram in product development

According to V-diagram, the product development starts from the requirements, conceptual design and system design. Researchers develop a lot of frameworks based on the System Model Language (SysML) on the level of system design $[2,5,6]$. These frameworks include different structures and seems to be not appropriate to all variety of real product cases. The one model based system modelling tool is LMS System Synthesis for now is actively developing [7]. System Synthesis is an architecture-driven approach for model-based systems engineering which allows engineers to reduce model design effort thanks to traceability and reusability of simulation models for different types of analyses such as requirements analysis or what if analysis. Models created in System Synthesis tool is linked with subsystems or functional model which may be created in LMS Amesim software or another tool. The architecture of a product is determined on the level of system design and after the choosing of architecture, it is proposed to apply optimization to narrow the limits of various parameters of the system. The example of a such approach will be presented in next sections of this paper. 
After the stages of system and functional level design, the component design of a product is performed. On the stage of component design the parametric or topology optimization of specific part of a product is usually applying. For example, for aircraft a lot of research is devoted to structural optimization [810], because the aircraft has high degree of reliability and at the same time should have a minimum weight. For all other products, it is important to apply optimization approach in order to reduce the number of testing and decrease time to market.

This paper aims to present optimization approach on different levels of product development based on the Unmanned Aerial Vehicle (UAV) development case study. UAV was developed according to V-diagram and optimization was performed to the numerical models of a UAV on system model to determine the angle of launch, on the functional level to determine the launch system parameters and on the component level to obtain structural parameters of a wing. The presentation of the material in the paper follows the sequence indicated above.

\section{Case study: tube-deployable small UAV}

Applying of optimization approach to the system level and structural design is considered on the example of tube-deployable small UAV, presented in the Fig. 2


Fig. 2. Concept of launch system and tube deployable small UAV

UAV has wings, which are may folded and unfolded. In initial state, when UAV is inside the tube the wings are folded. The pressure inside tube creates the force which acts to UAV. The force accelerates the UAV and it flies out of the tube (Fig. 2 left). Tube has a given angle of a slope. Then the wings open for a certain time and the plane gains altitude.

The UAV consists of four subsystems: engine, accumulator, launch subsystem and flight dynamics. The system model (Fig. 3) for UAV was developed in LMS System Synthesis software to describe how each subsystem works.

System model also includes four subsystems: Accumulator, Engine, Launch and Flight Dynamics. Accumulator generates required power, the engine transforms electric energy into mechanical energy, mechanical energy of a motor transforms to the ability of flight in flight dynamics subsystem, and Launch subsystem 
produces the mechanical energy required to fly out UAV from the tube. There are links between subsystems, which transmit energy in different forms. For UAV case study each subsystem was built in LMS Amesim software (Fig. 4).

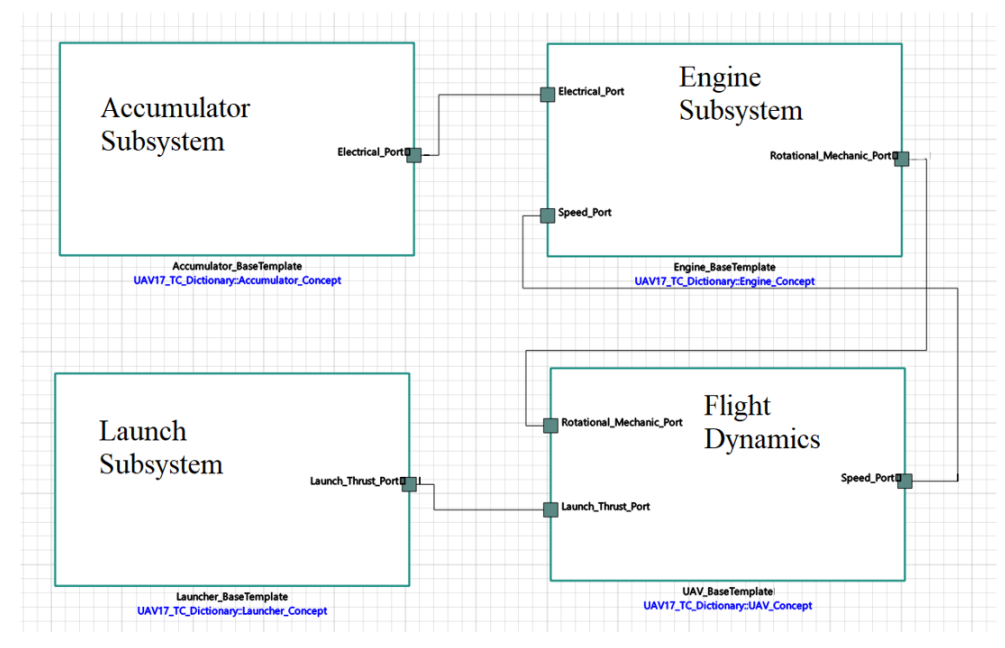

Fig. 3. System model for a small UAV in LMS System Synthesis software

\section{Architecture choice}

Different simulations were performed based on the system model. Simulation performed in System Synthesis solves the problem of architecture choice. Simulation allows to choose appropriate Engine Subsystem for UAV case study. Based on the simulation, the problem of appropriate angle of a launch system (Fig. 2) was solved. It is obvious, that the incorrect angle of a launch system can lead to the improper take off of the aircraft.

The altitude dependency over time for two architectures with different Engine subsystems is presented in Fig. 5. Two different engines are modeled, which are on the market. The difference between two engines lies in the coefficients, such as armature winding resistance and armature winding inductance.

The altitude climbing is better for Engine 1, and the 60 degree is a better angle for all architectures as seen from the figure 5 . Therefore, for further research architecture with Engine 2 was chosen. The next step is applying optimization to clarify the exact value of an angle to achieve maximum altitude.

\section{Optimization of a launch angle}

After choosing the rational variant of architecture by analyzing Fig. 5, it is required to calculate and set the optimal angle of launch in order to achieve 


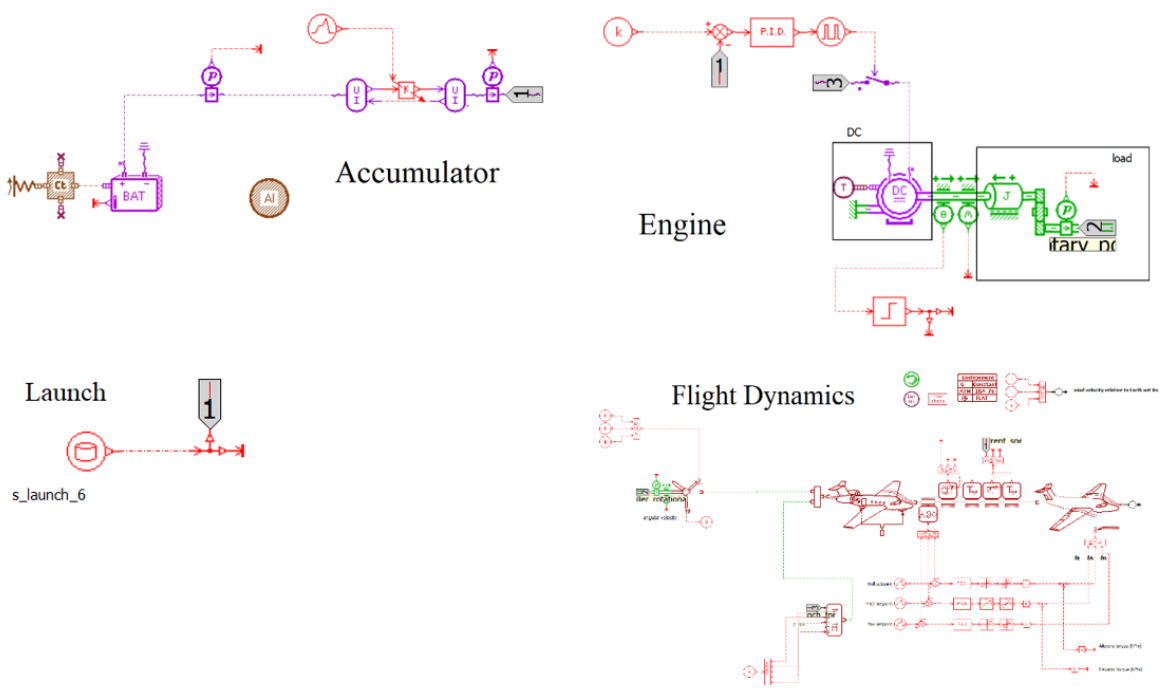

Fig. 4. Subsystems of a system model for a small UAV in LMS Amesim software



Fig. 5. Altitude dependency for two different engines and angles of launch 
maximum altitude and minimize fall in height after the point of maximum altitude, Fig. 5. Therefore, the statement of optimization problem can express as follows: to find launch angle which maximizes maximum altitude and minimize fall in height.

This problem was solved in Noesis Optimus software. The outline of optimization is presented on Fig. 6 .

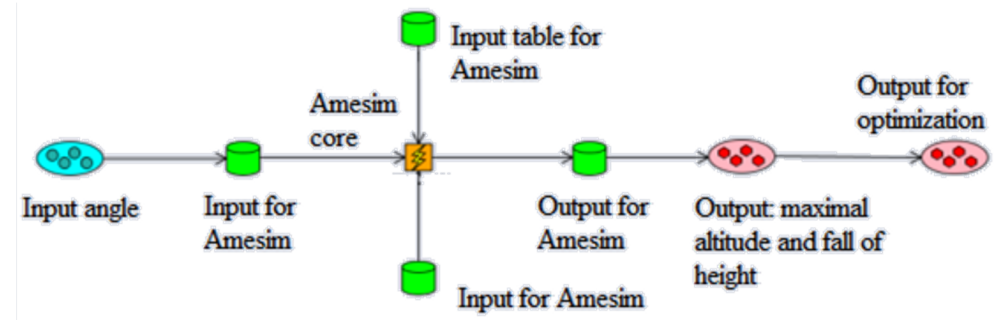

Fig. 6. Outline of optimization for search optimal angle

Blocks in outline are Input angle: the only angle, which varies from 40 to 80 degree; Amesim block, which has its own input and output blocks and the output block.

The results of optimization are presented below on Fig. 7 .

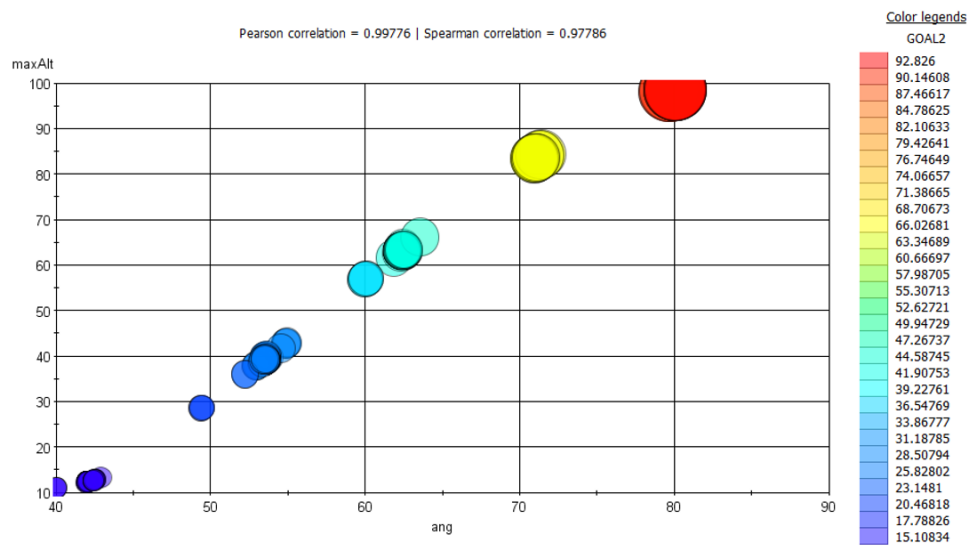

Fig. 7. Results of angle optimization

Fig. 7 shows the dependency of the maximal altitude over angle. The size and color of a bubble represent fall of height after the maximal altitude. Increasing the maximum height leads to an increase in the fall (Goal2 in Fig. 7). Thus, when the problem has two or more objectives, the researcher chooses a more 
appropriate variant. In this case, the optimal solution is 62 degree of the angle which leads to $63.3 \mathrm{~m}$ maximum altitude and $46.5 \mathrm{~m}$ of fall in height. It should be noted, that to avoid falling, it is necessary to work out the elevator.

This was the example of applying optimization on the system level and launch angle was determined as one of the important parameters in order to achieve better altitude gaining. Another example of applying optimization on the system level is parameters determination for the pneumatic tube.

\section{Optimization of parameters for a launch system}

The problem is to find better parameters for pneumatic tube (Fig. 2), which capable to provide flying out UAV and achieve for UAV a flight speed no less than $30 \mathrm{~m} / \mathrm{s} .30 \mathrm{~m} / \mathrm{s}$ is speed when UAV has appropriate lifting force.

A mathematical model for pneumatic tube was developed in LMS Amesim software to solve the indicated problem. The outline of a model is presented in Fig. 8.

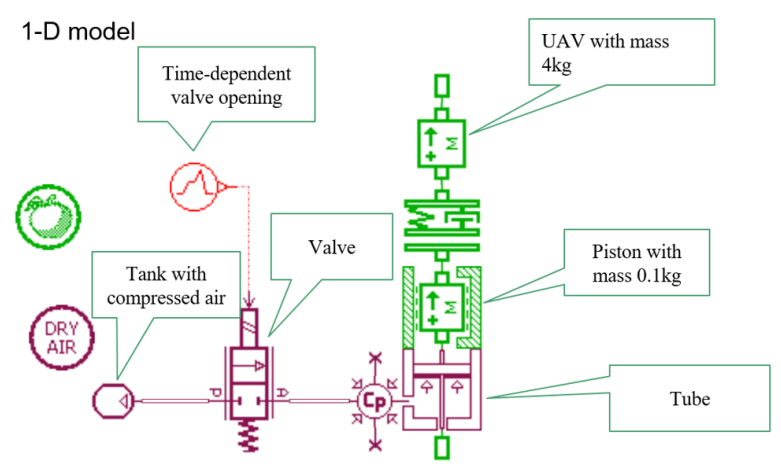

Fig. 8. 1-D model of a pneumatic launch system

Seven parameters need to be determined for presented model. Table 1 represents the list of these parameters and the limits of their variation.

Internal optimization tool of LMS Amesim software was chosen to solve the problem and Generic algorithm was the method of optimization. Optimal solution for this problem is on the Table 1 and some graphical results are presented in Fig. 9. They are length and pressure over iteration.

It should be noted that such a large number of iteration in the optimum search (Fig. 9) is caused by a large number of parameters, and by the choice of the genetic algorithm as an optimization method. Another appropriate optimization algorithms in LMS Amesim led to local minima, but a global one was required. This was the reason for choosing a long-term processing genetic algorithm.

Example above illustrates optimization on the functional level with help of specific optimization tool. It is not so crucial what tool to use for optimization 
Table 1. Table captions should be placed above the tables.

\begin{tabular}{|l|l|l|l|}
\hline Parameters & Lower boundary & Upper boundary & Optimal value \\
\hline Length of tube & $0.7 \mathrm{~m}$ & $2 \mathrm{~m}$ & $1.9 \mathrm{~m}$ \\
Tank volume & 2 liters & 10 liters & 9.9 liters \\
Tank pressure & 2 bars & 10 bars & 8.1 bars \\
Interior tube diameter & $20 \mathrm{~mm}$ & $100 \mathrm{~mm}$ & $48.1 \mathrm{~mm}$ \\
Valve opening time & $0.02 \mathrm{~s}$ & $0.1 \mathrm{~s}$ & $0.093 \mathrm{~s}$ \\
Valve interior diameter & $15 \mathrm{~mm}$ & $45 \mathrm{~mm}$ & $41.4 \mathrm{~mm}$ \\
Pipes interior diameter & $10 \mathrm{~mm}$ & $50 \mathrm{~mm}$ & $43.9 \mathrm{~mm}$ \\
\hline
\end{tabular}
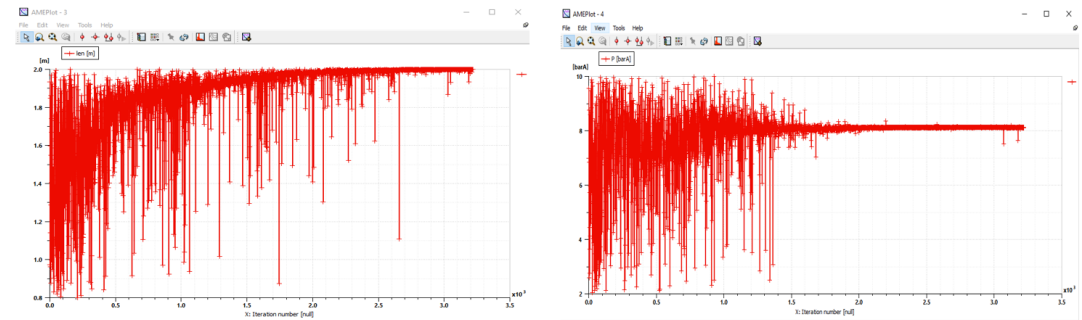

Fig. 9. Length of tube and pressure over iteration, during the optimization process

but it is very important to use the optimization approach for all significant numerical models of a system to accelerate the process of product design. The next section will be devoted to structural optimization of a wing.

\section{$6 \quad$ Structural optimization of UAV wing}

It should be noted, that for UAV case study the first prototype was produced from PLA plastic. Therefore, the parts were designed directly for 3D printing. The internal volume of the wing was filled with the honey structure as seen from Fig. 10.

In the wing, force structure is carbon spar. The plastic around the spar is the place where the spar is located. In this task, the mass of plastic around the spar was optimized. Fig. 11 represents force structure of a wing.

The input parameters for optimization are $\mathrm{L}$ and $\mathrm{R}$ (Fig. 11). $\mathrm{L}$ is the distance of increase the spar towards hole. $\mathrm{R}$ is fillet radius. There are two objectives in optimization: minimum of a displacement for the tip of force structure and minimum of a stress. The problem was solved in pSeven 6.12 software. Fig. 12 presents the results of optimization.

Fig. 12 shows results of optimization in a form, from which it can be seen the solution: maximal values of input parameters lead to minimal objectives. The values which lead to optimal solution are $\mathrm{L}=20 \mathrm{~mm}$, and $\mathrm{R}=20 \mathrm{~mm}$. Under these conditions the face of the composite spar approaches the center of the hole and minimum displacement and stress occur. 


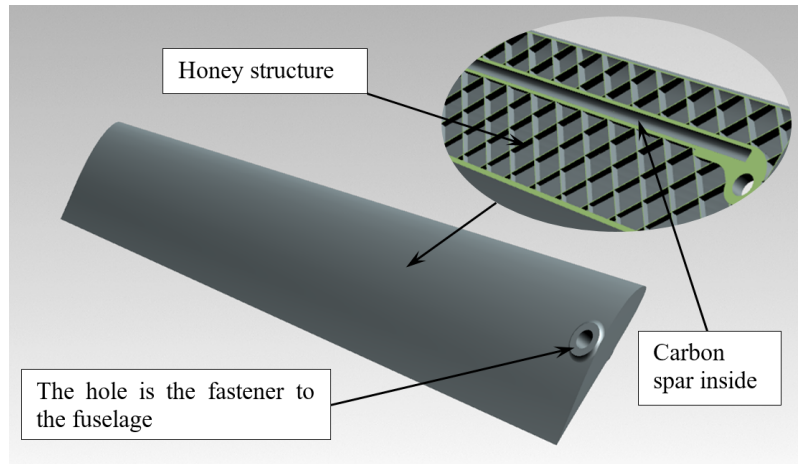

Fig. 10. Structure of a wing for small UAV



Fig. 11. Force structure inside the wing



Fig. 12. Parallel coordinates for input parameters L, R and for output maxDef, maxStress 


\section{Summary and outlook}

The central objective of the research was to demonstrate an integration of optimization approach with PLM and MBSE methodologies by means of applying optimization to small UAV case study. Meaning integration is followed: data in the form of numerical models is taken from PLM platform, then the important parameters are chosen and optimization is performed. Along with that, the requirements are checked and the optimization helps to meet these requirements in a faster way. Despite this tube deployable small UAV is not an industrial case and not all aspects of modeling and design of the UAV is presented in this paper, but optimization approach is supposed to be expanded towards the development of any innovative product. This approach implies optimization on the system, functional and structural numerical models over the process of product development and implementation of the optimization decreases the time of product development. The essence of the optimization approach technique is the appropriate choice of parameters and numerical models to optimize on each level of product development. Therefore, the combination of PLM, MBSE and Optimization approach creates strong methodology in which models are the core of product development, the optimization precise the models and the PLM allows to manage data efficiently.

\section{References}

1. Danesi, Frdric, et al. "P4LM: A methodology for product lifecycle management." Computers in industry 59.2-3 (2008): 304-317. https://doi.org/10.1016/j.compind.2007.06.013

2. Vosgien, Thomas, et al. "Towards model-based system engineering for simulationbased design in product data management systems." IFIP International Conference on Product Lifecycle Management. Springer, Berlin, Heidelberg, 2012. https://doi.org/https://doi.org/10.1007/978-3-642-35758-9 5

3. Fisher, Amit, et al. "3.1. 1 model lifecycle management for MBSE." INCOSE International Symposium. Vol. 24. No. 1. 2014. https://doi.org/https://doi.org/10.1002/j.2334-5837.2014.tb03145.x

4. Tahera, Khadija, Claudia Eckert, and Christopher Earl. "Highlighting the importance of testing in the product development process." (2015).

5. Peak, Russell S., et al. "9.3. 2 SimulationBased Design Using SysML Part 1: A Parametrics Primer." INCOSE international symposium. Vol. 17. No. 1. 2007.

6. https://www.plm.automation.siemens.com/en/products/lms/imagine-lab/systemsynthesis/

7. Rihtaric, J., R. avbi, and J. Duhovnik. "Sophytool for structural synhtesis of conceptual technical systems." DS 60: Proceedings of DESIGN 2010, the 11th International Design Conference, Dubrovnik, Croatia. 2010.

8. Chase, Nate, et al. "Structural Optimization of Composite Aircraft." 12th AIAA Aviation Technology, Integration, and Operations (ATIO) Conference and 14th AIAA/ISSMO Multidisciplinary Analysis and Optimization Conference. 2012.

9. Chedrik, Vasily, and Sergey Tuktarov. "Structural design of aircraft wing based on topology and global-local optimization." 11 th World Congress on Structural and Multidisciplinary Optimization.Sydney, Australia. 2015. 
10. Kachel, Stanislaw. "Optimization of wing parameters to achieve minimum weight at defined aerodynamic loads." Journal of Theoretical and Applied Mechanics 51 (2013). Oct 2017 